\title{
Surface roughness of ultra-thin silver films sputter deposited on a glass
}

\author{
Z. RAKOCEVIC*, R. PETROVIC $\dagger \&$ S. STRBAC $\ddagger$ \\ ${ }^{*}$ Laboratory of Atomic Physics, INS Vinca, P.O. Box 522, 11001 Belgrade, Serbia \\ $\dagger$ Institute of Physics, University of Belgrade, P.O. Box 57, 11001 Belgrade, Serbia \\ $\ddagger$ ICTM-Institute of Electrochemistry, University of Belgrade, P.O. Box 815, 11001 Belgrade, \\ Serbia
}

Key words. Glass, scanning tunnelling microscopy, silver, sputter deposition, surface roughness, ultra-thin films.

\begin{abstract}
Summary
Silver was sputter deposited on a glass with a thin film thickness ranging from 10 to $80 \mathrm{~nm}$. Scanning tunnelling microscopy was used to study the morphology of the obtained $\mathrm{Ag}$-glass surfaces and to estimate the surface roughness. An equation for the surface roughness of the thin film was evaluated using parameters related to the thin film features: the surface roughness of the substrate, the compressibility of the thin film and the film thickness. The experimental results were fitted using the evaluated equation, and the conditions favouring lower or higher surface roughness were analyzed.
\end{abstract}

\section{Introduction}

The growth of thin films of silver on a glass substrate has been widely studied using various methods. The morphology of the Ag thin films varied depending on the film thickness and the preparation procedure. For $\mathrm{Ag}$ thin films obtained by vapour deposition, significant differences in the topography of the films were obtained on varying the film thickness by changing the evaporation time (Van Duyne et al., 1993; Eberle et al., 2002; Nowakowski et al., 2002). For silver films thicker than 60 $\mathrm{nm}$, a preferred (111) orientation of the silver crystallites was assumed (Eberle et al., 2002). Studies of the influence of the deposition rate on the morphology of Ag thin films were also reported (Schlegel \& Cotton, 1991). For Ag thin films deposited on glass by radio frequency (rf) magnetron sputtering, it was found that the surface roughness increases with increasing film thickness (Kapaklis et al., 2006). A moderate(111) texture was found for thicker Ag films as well. In our earlier study on sputter deposited Ag thin films on glass, it was found that, at a constant deposition rate, the surface roughness exhibited a

Correspondence to: S. Strbac. Tel: +381 113370 389; fax: +381 113370 389; e-mail: sstrbac@tmf.bg.ac.yu minimum at a certain film thickness (Petrovic et al., 2001). In addition, it was shown that the surface roughness was dependent on the growth temperature, as well as on postdeposition heating of the sample (Van Duyne et al., 1993; Nowakowski et al., 2002; Lv et al., 2007).

In this work, the surface roughness and morphology dependence of silver thin films sputter deposited on a glass, on the deposition rate and on the deposition time were examined. On the basis of the obtained results, an equation for surface roughness of the thin film was evaluated using parameters related to the features of the thin film: the surface roughness of the substrate, the compressibility of the thin film and the film thickness.

\section{Methods}

Silver was sputter deposited onto highly polished glass substrates $(1 \mathrm{~cm} \times 1 \mathrm{~cm})$ (Glasco Products, Chicago). All depositions were performed at room temperature in a commercial rf diode sputtering vacuum system under an argon atmosphere. The residual pressure in the chamber was $3 \times 10^{-7}$ mbar, whereas the partial pressure of argon was $1 \times$ $10^{-3}$ mbar. Various deposition times and various deposition rates were exploited, thus producing samples of deposited thin films of silver with different thickness. The deposition rates were $0.0225,0.305,0.97,0.305$ and $2.6 \mathrm{~nm} \mathrm{~s}^{-1}$ (the error was lower than $4 \%$ ). For each deposition rate, the deposition times were chosen to produce samples with a thickness of the Ag thin film of 10, 20, 30, 40, 60 and $80 \mathrm{~nm}$. The thickness of the deposited silver films was measured using a profilometer Talystep 1, with an error lower than 5\%.

The surface morphologies of the 30 different samples of deposited Ag thin films were studied by an STM NanoScope III (Veeco Instruments Inc., Plainview, NY, USA) operating under ambient conditions. The images were obtained immediately 
a)

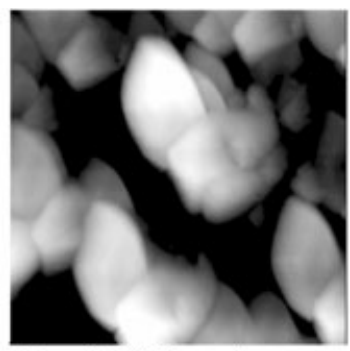

$\mathrm{R}=0.305 \mathrm{~nm} / \mathrm{s}$

b)

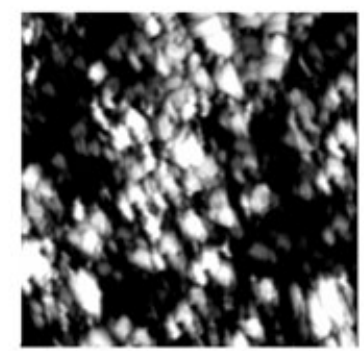

$\mathrm{R}=0.305 \mathrm{~nm} / \mathrm{s}$

c)

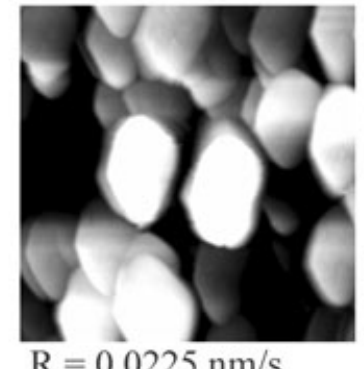

$\mathrm{R}=0.0225 \mathrm{~nm} / \mathrm{s}$

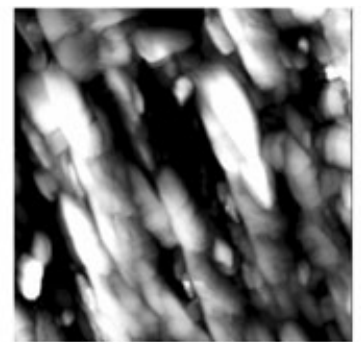

$\mathrm{R}=0.97 \mathrm{~nm} / \mathrm{s}$

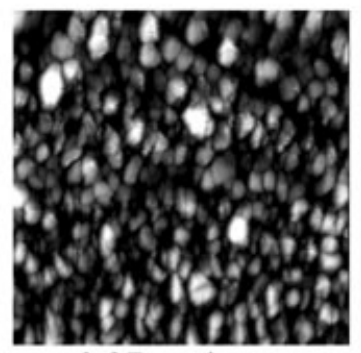

$\mathrm{R}=0.97 \mathrm{~nm} / \mathrm{s}$

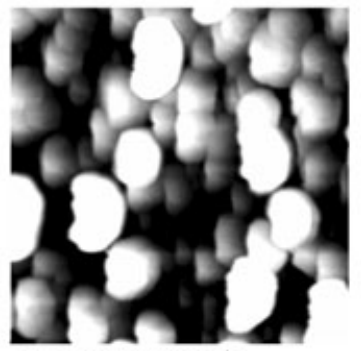

$\mathrm{R}=0.305 \mathrm{~nm} / \mathrm{s}$

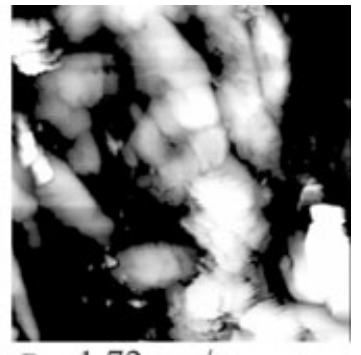

$\mathrm{R}=1.72 \mathrm{~nm} / \mathrm{s}$

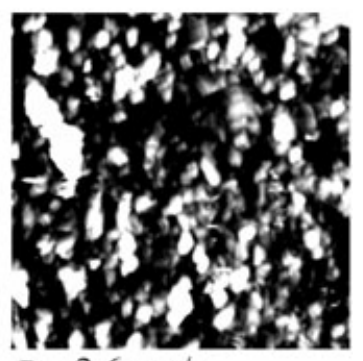

$\mathrm{R}=2.6 \mathrm{~nm} / \mathrm{s}$

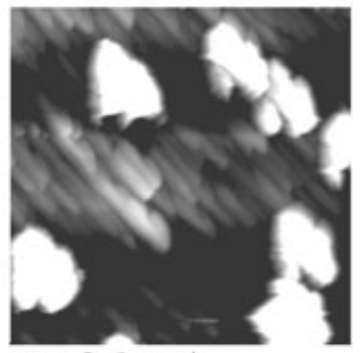

$\mathrm{R}=2.6 \mathrm{~nm} / \mathrm{s}$

Fig. 1. STM images of thin silver films deposited on a glass substrate with thicknesses of: (a) 10 , (b) 40 and (c) $80 \mathrm{~nm}$. The deposition rate used to obtain a particular sample is given below each image. All images were acquired at a scan size of $(880 \times 880) \mathrm{nm}^{2}$, and a $z$ range of $30 \mathrm{~nm}$, for each individual sample.

after each deposition by scanning over the area of $(880 \times$ 880) $\mathrm{nm}^{2}$ in the constant-current mode using a $W$ tip. The set-point current ranged from 7.1 to $8.5 \mathrm{nA}$, and the bias voltages from 16.4 to $18.7 \mathrm{mV}$, the $z$ range was at $30 \mathrm{~nm}$, and the lateral resolution at $(512 \times 512)$, the maximum number of data points provided by the instrument. For each sample, the surface roughness was estimated using the subprogram packages of the NanoScope III, as the average value of at least 10 STM images taken from different points within the substrate. The surface roughness is characterized by the roughness exponent, the lateral correlation length and the root mean square (rms) roughness, $H$, or the standard deviation of the surface height, $h: H \equiv\left|\left\langle h^{2}\right\rangle\right|^{1 / 2}$ (Tong \& Williams, 1994). The rms roughness is a quantitative value estimated from STM images with a maximum error of $5 \%$. The rms surface roughness is a function of the length scale over which it is measured. As the length scale expands, the roughness achieves a saturation value at the correlation length. Since for all the acquired images, the scan size of $880 \mathrm{~nm}$ exceeds the correlation length by a few hundred nanometres, the whole roughness spectrum was accessed in all cases.

\section{Results and discussion}

\section{Morphology of the Ag thin films deposited on glass}

The morphology of the deposited Ag thin films is presented by the STM images in Fig. 1, for thicknesses of 10, 40 and $80 \mathrm{~nm}$ and for various deposition rates. The change in the morphology with increasing film thickness is obvious, as is the change with deposition rate. For 10-nm-thick thin silver films (Fig. 1(a)), the deposit obtained using the lowest deposition rate consists of randomly distributed grains. The almost hexagonal shape of the deposited 200-250-nm large Ag grains indicates a crystalline structure of the (111) 


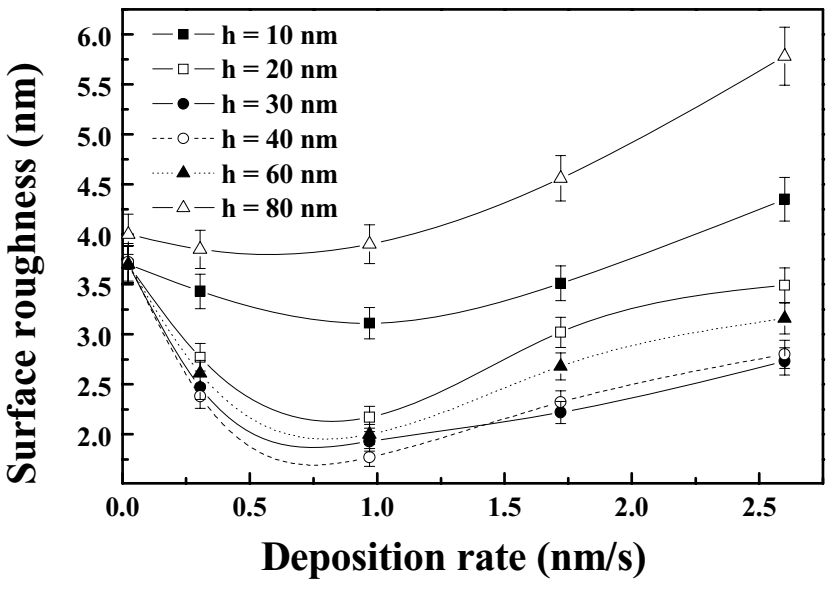

Fig. 2. The dependence of the average surface roughness on the deposition rate for various thicknesses of deposited silver films.

orientation. Grain size slightly decreases with the increase of the deposition rate, whereas the hexagonal shape of the grains disappears. Similar images were obtained for films with the thickness of 20 and $30 \mathrm{~nm}$ (not presented). For 40-nm-thick silver films (Fig. 1(b)), a quite different deposit morphology was obtained. Deposited silver grains obtained using the lowest deposition rate were much smaller, with the size ranging from 10 to $20 \mathrm{~nm}$, whereas the distribution of the grains over the surface was more uniform. With increasing deposition rate, the morphology did not change significantly. The lateral dimensions of the grains were almost identical, but their height increased leading to a structure corresponding to spongy layers for the highest deposition rate. With further increase of the film thickness to $80 \mathrm{~nm}$, the deposit morphology changed again, which is illustrated in Fig. 1(c). Deposited thin silver film obtained using the lowest deposition rate consists of about 150-200-nm large grains distributed orderly along one direction. The deposited Ag grains were homogeneous in size, and the grain shape was clearly hexagonal. The grain size continuously decreased with increasing deposition rate.

The average surface roughness was calculated from the STM images as described in the Methods section. Its dependence on the deposition rate for various thickness of the deposited film is shown in Fig. 2. For low deposition rates, the surface roughness first decreased with increasing deposition rate until it reached a minimum, and then increased again. The value of the surface roughness minimum depends on the film thickness. For films $10 \mathrm{~nm}$ thick, the surface roughness minimum was around $3.4 \mathrm{~nm}$. With increasing film thickness, this value decreased. Thus, for layers $40 \mathrm{~nm}$ thick, the surface roughness minimum was $1.7 \mathrm{~nm}$. On increasing the film thickness further, the value of the minimum increased. For films $80 \mathrm{~nm}$ thick, the minimum diminished. For higher film thickness, the surface roughness increased continuously with increasing deposition rate. Detailed analysis of the surface roughness minimum for the same system Ag-glass has already been reported by Petrovic et al. (2001). It was reported that the surface roughness minimum coincides to the turning point when the influence of the substrate becomes negligible, that is to the change in the nature of the system substrate-deposit from $\mathrm{Ag}$-glass to Ag-Ag. It can be noticed that the dependence of the average surface roughness and the average grain size on the deposition rate is similar. This indicates a direct relation between surface roughness and grain size for sputter deposited ultra-thin silver films.

\section{Surface roughness equation}

To derive an equation, which can explain above-described dependence of the surface roughness on the deposition rate, some real parameters characterizing the thin film behaviour have to be taken into account. According to the ballistic model of thin film growth (Family \& Vicsek, 1985), a deposited thin film consists of a certain number of monolayer that differs in the population of the atoms. The orderliness of the deposited film depends on the atom's population in each particular monolayer. Here, a new parameter, the average number of the unpopulated sites in a monolayer, $\bar{n}$, is introduced, which can be presented as

$$
\bar{n}=\frac{V K}{V_{\mathrm{ml}}}=\frac{S h K}{S d}=\frac{h}{d} K,
$$

where: $V$ is the volume of the deposited thin film, $K$ is its compressibility, that is the measure of its ability to compress, $V_{\mathrm{ml}}$ is the volume of a monolayer, $S$ is the surface area, $h$ is the thickness of the deposited thin film and $d$ is the monolayer thickness corresponding to the distance between two monolayers of the same orientation. In this equation, the compressibility is directly related to the average number of unpopulated sites in a monolayer $\bar{n}$. Taking into account that for ordered films, $K=0$, whereas for maximally disordered films, $K=1$. VK corresponds to the volume of the unpopulated sites taking the volume of a single atom as the volume unit.

Owing to the finite film thickness and the real surface roughness, the number of unpopulated sites in the subsequent monolayer, $n$, decreases going from the surface to the substrate and asymptotically tends to a constant value $n_{0}$ (the minimal number of unpopulated sites in a monolayer) (Fig. 3). For an ideally flat substrate surface (Fig. 3(a)) and a deposited film thick enough, the constant, $n_{0}$, is achieved at a certain depth ( $h=h_{1}$ ) depending on the nature of the deposit (the size of atoms or molecules, the type of the crystal lattice and the mobility of the deposited species) as well as on the deposition rate. For film thickness lower than $h_{1}\left(h<h_{1}\right)$, the change in the number of unpopulated sites in a monolayer with depth is directly proportional to the relative change in the film thickness:

$$
|\Delta n| \propto \frac{\Delta h}{h} .
$$

For a real substrate surface, which has its own roughness, a second characteristic thickness, $h_{0}$, has to be involved 


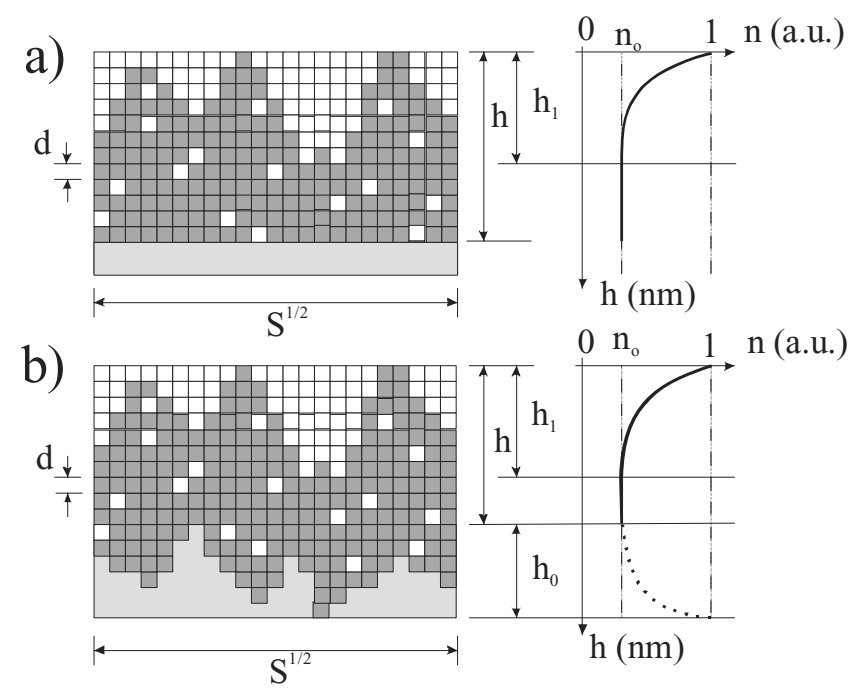

Fig. 3. Number of unpopulated atom sites, $n$, in a subsequent monolayer of the deposit: (a) for an ideally flat substrate surface and (b) for substrates with a real surface roughness. $h$ is the thickness of the deposited film, $h_{1}$ is the depth and $h_{0}$ is the distance from the substrate surface, where a constant number of unpopulated sites, $n_{0}$, is achieved.

(Fig. 3(b)). Namely, starting from the other side of the film, that is from the substrate, a thin film also exhibits a changeable number of unpopulated sites in the subsequent monolayer. The number of unpopulated sites in the deposit caused by the roughness of the substrate also decreases asymptotically up to a constant value, $n_{0}$ (the bulk of the deposit), which is achieved at a distance $h_{0}$ from the minimum of the surface roughness of the substrate (see the dotted part of the curve in Fig. 3(b)).

According to the considerations given earlier, it can be seen that the surface roughness is mainly determined by the part of the deposited film with the thickness $h_{1}$. The bulk of the thin film has an influence on the roughness only for higher values of the constant $n_{0}$, that is if there is a higher number of unpopulated sites. For a low film thickness, $h_{1}$ and $h_{0}$ might overlap, which would lead to a more complicated dependence of the surface roughness on the thickness of the film. For the other cases, an equation describing the dependence of the surface roughness on the film thickness can be evolved as described later.

By solving Eq. (2) with respect to film thickness, $h$, and by taking the rms, Eq. (3) is obtained:

$$
H=H_{01} \exp (\text { const } 1 \bar{n}),
$$

where $H$ is the rms surface roughness; $H_{01}$ is the roughness of the deposited film for $\bar{n}=n_{o}$ at a distance $h<h_{1}$ and $h>h_{0}$ (see Fig. 3(b)); $\bar{n}$ is described earlier and const 1 depends on the nature of the deposit.

By contrast, for the roughness of the substrate surface, $H_{0}$, a similar equation can be obtained

$$
H_{o}=H_{o 1} \exp (\text { const } 2 \bar{n}),
$$

where the parameters $H_{01}$ and $\bar{n}$ remain the same as described earlier, whereas const 2 is different and depends on the nature of the substrate-deposit. Substituting $H_{01}$ from Eq. (4) into Eq. (3), one obtains

$$
H=H_{o} \exp (\operatorname{const} \bar{n}),
$$

where $\mathrm{H}$ and $H_{0}$ are the roughness of the deposited film and of the substrate, respectively, and const depends on the nature of the system.

Substituting $\bar{n}$ from Eq. (1) into Eq. (5), the expression for the surface roughness as a function of the compressibility, $K$, and the film thickness, $h$, is obtained

$$
H=H_{o} \exp \left(\operatorname{const} \frac{h}{d} K\right) .
$$

It is well known that surface roughness is generally counterbalanced by a smoothing or ordering process owing to the mobility of the deposited atoms (Meakin, 1993; Rakocevic et al., 1995). The compressibility, defined earlier as the ability of the film to compress, represents an overall measure of the mobility of the atoms (Gomer, 1990). At a constant temperature, it is defined as the average square of the fluctuation of the number of atoms per unit surface area. There are two preferred directions in the mobility of atoms: one perpendicular to the surface and the other along the surface. These directions exhibit asymmetry in the vertical and horizontal mobility of atoms. Along the surface, there is a certain density of atoms, $Q_{\mathrm{H}}$, exhibiting a horizontal diffusion, contributing to an increase in the orderliness of a layer and consequently to a decrease in compressibility. Also, there is a certain density of atoms exhibiting a vertical diffusion, normal to the surface (the inter-layer diffusion), $Q_{V}$, which also contributes to an increase in the orderliness of a layer. In addition, the depositing atoms arriving on the surface from a vertical direction (which in a time unit corresponds to the deposition rate, $R$ ) contribute to a decrease in the orderliness of the deposited layer and consequently to an increase in compressibility. The compressibility can thus be expressed as

$$
K \propto \frac{\left\langle\left(R-Q_{V}\right)^{2}\right\rangle}{\left\langle Q_{H}^{2}\right\rangle} .
$$

The compressibility defined in such a way can have values 0 $\leq K<1$. An ideally ordered film, $K=0$, is obtained when the horizontal diffusion is high and $Q_{V} \neq R$, whereas an ideally disordered film, $K \rightarrow 1$, is obtained when $Q_{\mathrm{H}}+Q_{\mathrm{V}} \rightarrow R$.

Substituting $K$ from Eq. (7) into Eq. (6) and assuming that, to a first approximation, most of the depositing atoms diffuse only horizontally, which means that $R^{2} \gg Q_{V}^{2}$ and consequently that $Q_{V}^{2}$ can be neglected. The surface roughness of a film can be expressed as

$$
H=H_{o} \exp \left[C h \frac{R^{2}-2 Q_{V} R}{Q_{H}^{2}}\right],
$$

where $C$ is a constant; the other values are as explained earlier. From this equation, it can be seen that the surface roughness 
can be expressed as a function of the deposition rate $R$, the density of atoms diffusing vertically, $Q_{\mathrm{V}}$, and the density of atoms diffusing horizontally, $Q_{\mathrm{H}}$.

Fitting the surface roughness equation with the experimental data

The above-given Eq. (8) was applied to the experimental results presented here for the deposition of $\mathrm{Ag}$ on glass. The results obtained by fitting Eq. (8) and the experimental data from Fig. 2 are presented in Fig. 4. In the range of experimental error, these results show a reasonable agreement. For simplicity, only the results for three film thicknesses are presented.

Equation (8) can also be applied to estimate the vertical and horizontal atom diffusion as a function of film thickness, which is presented in Fig. 5. The values were calculated with an error of $10 \%$. The value of the constant $C$ was obtained on the basis of the following consideration. The dependence of $Q_{H} / \sqrt{C}$ on the film thickness (up to a film thickness of $40 \mathrm{~nm}$ ) is linear

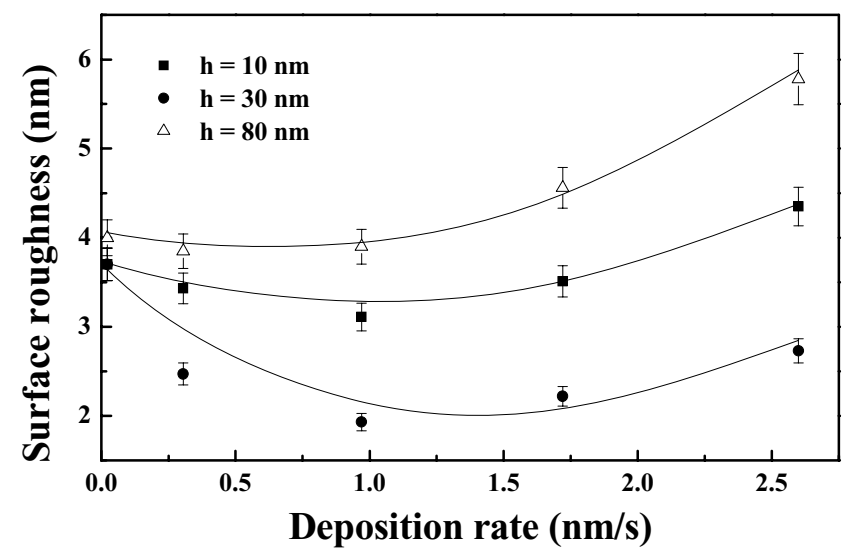

Fig. 4. The dependence of the surface roughness on the deposition rate for various film thicknesses as obtained by fitting. The spots represent the experimental data.

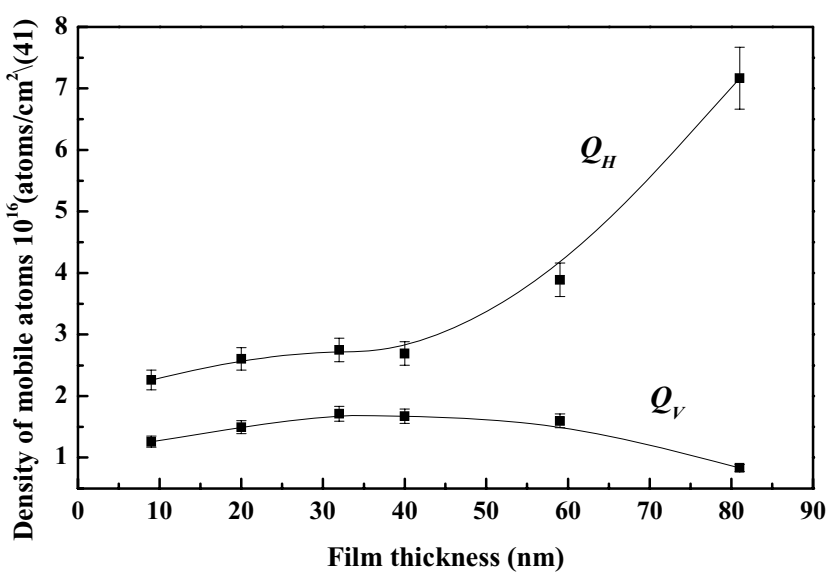

Fig. 5. The dependence of the density of atoms diffusing horizontally, $Q_{H}$, and vertically, $Q_{\mathrm{V}}$, on the thickness of the silver film. with an intercept at the $y$-axis (not presented) corresponding to the density of atoms diffusing over an ideally flat surface $(h=0)$. Since the substrate is not ideally flat, but has its real roughness, $H_{0}$ (in this case $3.7 \mathrm{~nm}$ ), the value of the intercept has to be corrected. To obtain an ideally flat substrate, half of the volume $\left(1 \mathrm{~cm}^{2} \mathrm{H}_{0}\right)$ has to be filled with deposited atoms. These atoms correspond to the maximum number of atoms that can participate in surface diffusion (assuming that for $h$ $=0$, only horizontal diffusion occurs). From the ratio between the intercept and the maximum number of diffusing atoms for a real surface, the constant $C$ can be calculated $(C=0.055$ $\mathrm{nm}^{-1}$ ).

The obtained dependences for the horizontal and vertical atom diffusion showed different behaviour depending on the film thickness. For films from 10 to $40 \mathrm{~nm}$ thick, the density of atoms diffusing horizontally linearly increased. After the thickness of $40 \mathrm{~nm}$ is achieved, this density of atoms increased sharply. The density of atoms diffusing vertically changed in a different way. Starting from the lowest film thickness, the mobility increased, reached a maximum for a film thickness from 30 to $40 \mathrm{~nm}$, after which it decreased, tending to a negligible value. Thus, for all films examined, the density of atoms exhibiting horizontal mobility was higher than the density of atoms exhibiting vertical mobility: two to three times for low film thicknesses (10-40 nm) and six to seven times for higher film thicknesses $(80 \mathrm{~nm})$.

For low film thickness, the formation of $2 \mathrm{D}$ clusters is more likely, involving the horizontal atom diffusion that, as a consequence, exhibits a linear dependence of surface diffusion on the film thickness. With increasing film thickness, the growth and formation of 3D clusters occur, leading to an increase of the density of atoms exhibiting vertical diffusion. When the 3D clusters achieve a certain size, the density of atoms exhibiting vertical diffusion decreases sharply, whereas the density of atoms exhibiting horizontal diffusion increases. This can be explained by the formation of grains, and when the surface of these grains becomes large enough, new-arriving depositing atoms can move, collide and form 2 D clusters on top of the grains.

It was mentioned previously that the condition necessary to obtain an ideally ordered film $(H=0)$ is the zero compressibility. According to Eq. (7), this means that the deposition rate is equal to the density of atoms diffusing vertically. Taking this into account, after a certain film thickness has been achieved (see Fig. 5), $Q_{V}$ tends to zero, meaning that some other condition for obtaining an ideally ordered layer has to be considered. According to Eq. (8), this condition is that the density of atoms diffusing horizontally is much higher than the deposition rate, that is $Q^{2}{ }_{H}>>C h R^{2}$. This can be achieved by increasing the substrate temperature (Mandelbrot, 1982). The other way is that the material is deposited at near-grazing angles towards the substrate (Vicsek, 1989). By deposition in such a manner, horizontal diffusion of atoms is significantly increased. 


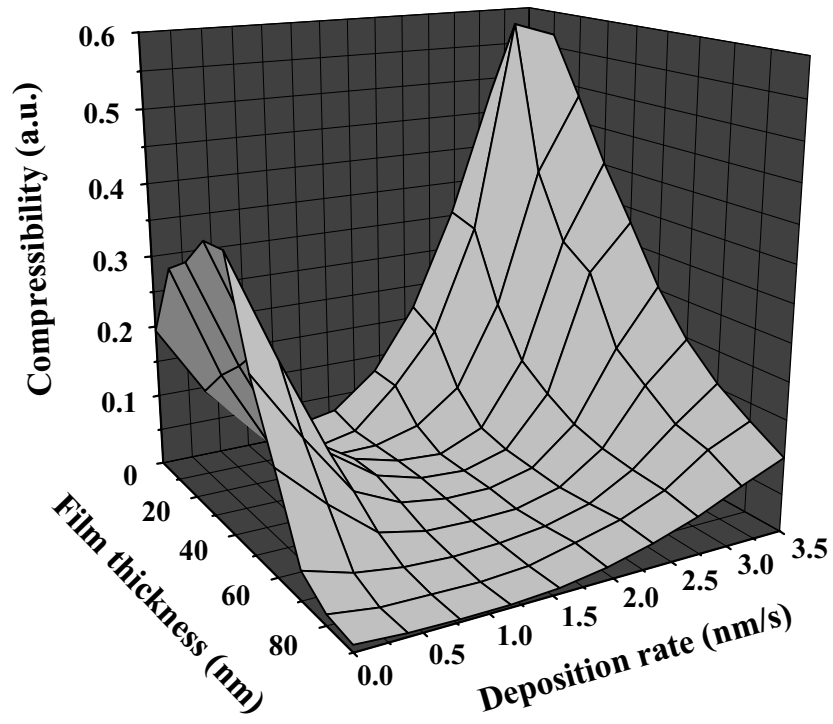

Fig. 6. The dependence of the average compressibility on the deposition rate and on the thickness of the silver film.

The dependence of the average compressibility on the deposition rate and on the film thickness is presented in a threecoordinate system in Fig. 6 . The compressibility is a complex function with respect to the deposition rate and the thickness of the thin film. This is the consequence of the overlapping of the influence of the substrate and thin film roughness (see Fig. 3(b)) and of the change in the nature of the system substrate-deposit from $\mathrm{Ag}-$ glass to $\mathrm{Ag}-\mathrm{Ag}$ at a certain film thickness.

\section{Conclusion}

An equation for the roughness of the thin film was evaluated. By fitting the experimental results using this equation, the parameters obtained were discussed with respect to the conditions necessary to favour lower or higher surface roughness.

\section{Acknowledgement}

We gratefully acknowledge the financial support by the Ministry of Science of the Republic of Serbia, project no. 06141001.

\section{References}

Eberle, R., Kresser, T. \& Pietralla, M.M. (2002) Elastic properties of thin silver films: hexagonal model and influence of corrugation effects. Thin Solid Films 408, 69-175.

Family, F. \& Vicsek, T. (1985) Scaling of the active zone in the Eden process on percolation networks and the ballistic deposition model. J. Phys. A. 18(2), L75-L82.

Gomer, R. (1990) Diffusion of adsorbates on metal surfaces. Rep. Prog. Phys. 53, 917-1002.

Kapaklis, V., Poulopoulos, P., Karaoutsos, V., Manouras, Th. \& Politis, C. (2006) Growth of thin Ag films produced by radio frequency magnetron sputtering. Thin Solid Films 510, 138-142.

Lv, J., Lai, F., Lin, L., Lin, Y., Huang, Z. \& Chen, R. (2007) Thermal stability of Ag films in air prepared by thermal evaporation. Appl. Surf. Sci. 253(17), 7036-7040.

Mandelbrot, B.B. (1982) The Fractal Geometry of Nature. Freeman, New York.

Meakin, P. (1993) The growth of rough surfaces and interfaces. Phys. Rep. 235(4-5), 189-289.

Nowakowski, R., Pielaszek, J. \& Dus, R. (2002) Surface mediated Ag$\mathrm{Hg}$ formation under ambient and vacuum conditions-AFM and XRD investigations. Appl. Surf. Sci. 199, 40-51.

Petrovic, R., Strbac, S., Bundaleski, N. \& Rakocevic, Z. (2001) Surface roughness minimum: Ag thin layer deposited on a glass. J. Serb. Chem. Soc. 66(7), 483-490.

Rakocevic, Z., Strbac, S., Bibic, N. \& Nenadovic, T. (1995) Surface diffusion of clusters and thin film growth. Surf. Sci. 343, 247-260.

Schlegel, V.L. \& Cotton, T.M. (1991) Silver-island films as substrates for enhanced Raman scattering: effect of deposition rate on intensity. Anal. Chem. 63, 241-247.

Tong, W.M. \& Williams, R.S. (1994) Kinetics of surface growth: phenomenology, scaling, and mechanisms of smoothening and roughening. Annu. Rev. Phys. Chem. 45, 401-438.

Van Duyne, R.P., Hulteen, J.C. \& Treichel, D.A. (1993) Atomic force microscopy and surface-enhanced Raman spectroscopy. I. Ag island films and Ag film over polymer nanosphere surfaces supported on glass. J. Chem. Phys. 99, 2101-2115.

Vicsek, T. (1989) Fractal Growth Phenomena. World Scientific, Singapore. 